Acta Cryst. (2002). A58 (Supplement), C261

SUB-SECOND X-RAY DIFFRACTION MEASUREMENT AND STRUCTURE ANALYSIS USING MSGC

H. Uekusa $^{1}$ Y. Ohashi ${ }^{1}$ A. Ochi ${ }^{2}$ T. Tanimori ${ }^{3}$

Tokyo Institute of Technology Department of Chemistry Ookayama 2, Meguro-Ku TOKYO 152-8551 JAPAN

${ }^{1}$ Department of Chemistry and Materials Science, Tokyo Institute of Technology, JAPAN ${ }^{2}$ Department of Physics, Kobe University ${ }^{3}$ Department of Physics, Kyoto University

Rapid X-ray diffraction measurement technique is an essential to realize the time-resolved observations of crystalline-state reactions, unstable species in excited state and reaction intermediates. To achieve time resolution of seconds or sub seconds order, the use of photon counting type two-dimensional detector is required. Recently, MSGC (Micro Strip Gas Chamber) was developed for the rapid X-ray data collection. MSGC is a gaseous detector having both photon counting and two-dimensional imaging properties. The features of the detector are a large detective area of $10 \times 10 \mathrm{~cm}$, a fine positional resolution of 100 micrometer and an excellent capability for high counting rate up to $10^{7} \mathrm{cps}$ suitable for the synchrotron radiation experiments. With the measurement system consists of a MSGC detector, fast-rotation one axis goniometer and a laboratory X-ray generator, complete three dimensional reflection data set from continuously rotating crystals of typical organic compounds were recorded within several minutes to seconds range and the results of structure analyses were satisfactory. Also, for an application of monitoring the diffraction pattern, the timing resolution of sub-second can be achieved in the observation of the change of cell dimensions caused by the excited molecules in the crystal. In the experiment using the same system with synchrotron radiation at BL46XU in SPring-8, crystal structure analysis of sub-second measurement data was successfully achieved to show the MSGC system is suitable for a time-resolved measurement using synchrotron radiation.

Keywords: MSGC, TIME-RESOLVED MEASUREMENT, SYNCHROTRON RADIATION

Acta Cryst. (2002). A58 (Supplement), C261

\section{FINDING THE OPTIMAL CONDITIONS FOR DATA COLLECTIONS USING THE NONIUS KAPPACCD}

\section{H. O. Sorensen S. Larsen}

Centre for Crystallographic Studies, University of Copenhagen Chemical Lab. IV Universitetsparken 5 COPENHAGEN 2100 DENMARK

Choosing the best strategy to measure the most accurate diffraction data is a very important issue in crystallography. How do we obtain the highest possible data quality with the time frame on hand? This even more so when collecting data for charge density studies, where a high precision is needed for data measured to high diffraction angles. A suitable crystal diffracting out to 1.12 $\AA^{-1}$ of tetrafluoroterephthalonitrile was chosen as the test system. Five data sets were collected on a Nonius $\kappa \mathrm{CCD}$ at $122(1) \mathrm{K}$ using MoKa radiation varying scan time and scan width to investigate the influence on the data quality. The following scan times per frame and scan widths were used; 1) $70 \mathrm{~s} /{ }^{\circ}, 1.00^{\circ}$; 2) $70 \mathrm{~s} /{ }^{\circ}, 0.50^{\circ}$; 3) $140 \mathrm{~s} / /^{\circ}, 0.50^{\circ}$; 4) $70 \mathrm{~s} /{ }^{\circ}, 0.25^{\circ}$; 5) $140 \mathrm{~s} /{ }^{\circ}, 0.25^{\circ}$. EvalCCD $[1]$ was used to integrate the collected data and equivalent reflections were merged in Sortav[2]. In EvalCCD a number of parameters (shoe box size and crystal description) controlling the integration procedure have furthermore been varied to study their influence on the quality as well as redundancy in the data set. The validation of the data quality in this study is based on the internal residuals, the number of reflections obtained, and their standard uncertainties.

[1] A. J. M. Duisenberg (1998). PhD thesis, University of Utrecht, The Netherlands. [2] R.H. Blessing (1987). Cryst. Rev., 1, 3.

Keywords: DATA COLLECTION STRATEGY NONIUS KAPPACCD DATA REDUCTION
Acta Cryst. (2002). A58 (Supplement), C261

HIGH RESOLUTION X-RAY DIFFRACTION WITH SIMPLE INTERMETALLIC COMPOUNDS

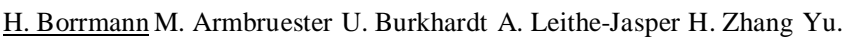
Grin

Max Planck Institute for The Chemical Physics of Solids Noethnitzer Str. 40 DRESDEN 01187 GERMANY

A three-circle goniometer in combination with a large imaging plate detector in Weissenberg type arrangement provides an excellent basis for fast data aquisition aiming both at high resolution as well as high redundancy. First results obtained for simple intermetallic compounds such as $\mathrm{CuAl}_{2}$ and $\mathrm{AlB}_{2}$ will be presented. Most notably the very high data to parameter ratio (> 80) from data collected out to $2 \theta_{\max }=140^{\circ}$ applying $\mathrm{MoK} \alpha$ radiation, offers excellent possibilities to use various refinement models but even allows for the refinement of correlated parameters, e.g. site occupancies. Special consideration is necessary for the $\mathrm{K} \alpha 1 / \mathrm{K} \alpha 2$ splitting at high diffraction angles. Accordingly, possibilities for single wavelength arrangements have been investigated.

\section{Keywords: INTERMETALLIC COMPOUNDS HIGH RESOLUTION IMAGING PLATE}

Acta Cryst. (2002). A58 (Supplement), C261

NEW HARDWARE AND TOOLS FOR MACROMOLECULAR CRYOCRYSTALLOGRAPHY

C. Bauer M. Benning B. He R. Durst M. Li

Bruker AXS 5465 E. Cheryl Parkway MADISON WI 53711 USA

The discovery that protein crystals could be successfully flash-frozen brought about a revolution in macromolecular crystallography. Today, the cooling of macromolecular crystals to liquid nitrogen temperatures has become a requirement during data collection under most circumstances. Despite this fact, freezing, storing, handling, and maintaining sample temperature during data collection can still be a cumbersome task, especially for novice users. In addition, the wide variation in experimental hardware designs that exists at various synchrotron beamlines and in home laboratory equipment makes the use of a standard protocol for crystal manipulation difficult. This presentation will focus on recent developments in hardware, tools, and new methods recently developed by Bruker AXS to help solve these difficult problems.

\section{Keywords: MACROMOLECULAR CRYOCRYSTALLOGRAPHY} METHODS 